\title{
Menstrual Cycle Regularity
}

National Cancer Institute

\section{Source}

National Cancer Institute. Menstrual Cycle Regularity. NCI Thesaurus. Code C156587.

A description of how an individual's menstrual cycle conforms to a consistent temporal pattern. 